\title{
Modelos de Equações Estruturais em Psicologia: Conceitos e Aplicações
}

\author{
Ronaldo Pilati ${ }^{1}$ \\ Jacob Arie Laros \\ Universidade de Brasília
}

\begin{abstract}
RESUMO - O debate sobre procedimentos e técnicas de tratamento de dados é relevante para o contexto de produção e avanço do conhecimento em várias áreas do saber. Os Modelos de Equações Estruturais são um conjunto de técnicas de tratamento de dados que têm recebido grande atenção de pesquisadores, especialmente nos últimos 10 anos. Esse procedimento de tratamento de dados possui suas raízes relacionadas a distintas áreas do conhecimento: a biometria, a econometria e a psicometria. Como conseqüência de suas peculiaridades, o relato científico em Modelos de Equações Estruturais deve considerar vários aspectos relevantes, como a definição teórica do modelo a ser testado, a especificação e identificação do modelo, além de aspectos de estimação e mensuração dos índices de adequação. Vários desses elementos são apresentados e discutidos no presente artigo focando-se nas possibilidades de aplicação na Psicologia e em ciências correlatas. Perspectivas futuras e limitações dos Modelos de Equações Estruturais são discutidas.
\end{abstract}

Palavras-chave: modelos de equações estruturais; análise de dados multivariados; modelos de equações múltiplas.

\section{Structural Equation Modeling in Psychology: Concepts and Applications}

\begin{abstract}
The debate concerning procedures and techniques of data treatment is relevant in the context of production and progress of knowledge in a broad range of subject areas. In the past decade, a framework of data treatment methods denominated Structural Equation Modeling received a great deal of attention from researchers. This data treatment procedure has its historical origins related to different areas of knowledge like biometrics, econometrics and psychometrics. As a consequence of its unique characteristics, scientific report in Structural Equation Modeling should consider various relevant aspects, like the theoretical definition of the model to be tested, the specification and identification of the model, as well as estimation and measurement aspects of the adequation indices. Several of these elements are presented and discussed in the present article, focusing on the possibilities of practical application in the field of Psychology and related sciences. In addition, future perspectives and limitations of Structural Equation Modeling are also discussed.
\end{abstract}

Key words: structural equation modeling; multivariate data analysis; multiple equation models.

Procedimentos de análise de dados são característicos na pesquisa em psicologia. A complexidade dos fenômenos estudados exige que os pesquisadores das diferentes áreas da psicologia desenvolvam ou apliquem técnicas multivariadas de análise de dados condizentes com os fenômenos estudados. Isso pode ser observado desde o desenvolvimento de modelos de testagem psicológica ao final do século XIX e princípios do século XX (Anastasi \& Urbina, 2000; Laros, 2005; Thompson, 2004), até as discussões mais recentes sobre delineamentos de pesquisa experimental e quase-experimental em Psicologia (Shadish, Cook \& Campbell, 2002).

Paralelamente ao desenvolvimento da pesquisa psicológica, nas duas últimas décadas, o desenvolvimento da microinformática tornou o uso e aplicação de modelos estatísticos mais complexos acessíveis aos pesquisadores em Psicologia.

1 Endereço: Departamento de Psicologia Social e do Trabalho, Instituto de Psicologia. Campus Universitário Darcy Ribeiro, Instituto Central de Ciências - Ala Sul, Brasília, DF, Brasil 70900-000.E-mail: rpilati@ unb.br
Isso ocorre graças ao aumento crescente da capacidade de processamento de computadores e programas estatísticos mais poderosos e de fácil manuseio.

Esse incremento popularizou o uso de análise estatística multivariada, o que permite o desenvolvimento teórico e metodológico do campo, possibilitando testes empíricos de modelos teóricos de forma cada vez mais completa, pois os modelos multivariados atuais permitem que dezenas de relações múltiplas possam ser testadas simultaneamente, o que é coerente com a complexidade dos fenômenos de interesse dos cientistas psicólogos. Mas, de qualquer forma, esses procedimentos de análise de dados exigem atenção e cuidado do pesquisador, para que ele seja capaz de realizar as aplicações corretas, bem como interpretar resultados adequadamente, evitando falácias ou falsas conclusões.

Uma das técnicas que tem se proliferado ultimamente na ciência psicológica e áreas correlatas são os modelos de equações estruturais (MEE). Essa técnica é entendida por autores do campo (e.g. Klem, 2002; Thompson, 2002; Ullman, 2007) como uma mistura de análise fatorial e análise de regressão, que permite aos pesquisadores testar estruturas fatoriais de instrumentos de medida psicométrica, por meio 
da análise fatorial confirmatória. A MEE não apenas permite o teste confirmatório da estrutura psicométrica de escalas de medida mas também pode ser utilizada para analisar relações explicativas entre múltiplas variáveis simultaneamente, sejam essas latentes ou observadas. Segundo Hox e Bechger (1998), a MEE proporciona um arcabouço geral e conveniente de procedimentos de análise de dados que incluem vários procedimentos tradicionais de análise de dados multivariados.

A nomeação da técnica é profusa, pois vários nomes diferentes podem ser observados na literatura. Nunally e Bernstein (1994) dizem que a técnica é conhecida como análise de estruturas de covariância e relações estruturais lineares. Já Ullman (2007) aponta que a técnica é também conhecida como modelagem causal, análise causal, modelagem de equações simultâneas e análise de estruturas de covariâcia. Como forma de nomear a técnica sem provocar maior profusão, adota-se, no presente trabalho, o termo modelos de equações estruturais.

Dentro do escopo internacional de produção de conhecimento em Psicologia, o uso dessa técnica de análise de dados tem se tornado cada vez mais comum. Em uma simples busca por operadores booleanos, restrita aos anos de 1980 a 2005, dentro de uma das mais abrangentes bases de dados de produção científica em psicologia (PsycLIT da American Pscyhological Association), obtém-se como resultado aproximadamente 1.400 artigos científicos que de alguma forma fazem uso da técnica de modelos de equações estruturais como mecanismo de tratamento de dados.

A grande profusão do uso dessa técnica de análise de dados também pode ser notada pelo intenso debate que se dá ao redor desse tema. Desde 1993, a SEMNET (Structural Equation Modeling Network), uma lista de discussão multidisciplinar dedicada a debater os tópicos de interesse de pesquisadores e desenvolvedores dos modelos matemáticos de MEE, está em funcionamento, contando, já em 1998, com mais de 1.500 integrantes de dezenas de países (Kaplan, 2000). Dentro dessa mesma perspectiva foi criado o periódico científico "Structural Equation Modeling: A Multidisciplinary Journal', que desde 1994 vem publicando artigos inéditos, empíricos ou teóricos que sejam relacionados ao tema de MEE. Como pode ser observado, a profusão do uso dessa técnica, bem como dos problemas e desafios decorrentes da mesma, são intensamente debatidos na produção internacional em Psicologia e outras áreas correlatas.

No contexto brasileiro, desde meados da década passada começou-se a notar um aumento de interesse sobre o uso da técnica de MEE como estratégia de análise de dados e teste de modelos teóricos. Em uma busca com operadores booleanos, na base de dados multidisciplinares Scielo, encontrou-se oito artigos que abordam o tema de MEE. Quando essa pesquisa é expandida para uma área correlata à Psicologia, a Administração, mais especificamente nos anais dos ENANPADs (Encontro da Associação de Pós-Graduação e Pesquisa em Administração) e resumos da RAC (Revista de Administração Contemporânea), RAE (Revista de Administração de Empresas) e RAUSP (Revista de Administração da USP) uma busca simples revela cerca de 25 trabalhos que utilizam a MEE como procedimento de análise de dados. Observa-se, graças a esses e outros indicadores, o início do interesse de pesquisadores brasileiros sobre a temática.
Uma das questões centrais sobre o uso acadêmico de modelos inovadores de tratamento de dados diz respeito à formação de pesquisadores e alunos de pós-graduação. A profusão de programas de análise de dados que realizam procedimentos estatísticos complexos não garante que cuidados essenciais no uso da técnica sejam observados, o que pode levar a resultados espúrios e mal interpretados, construção errônea de modelos, decisões sobre índices e pressupostos equivocadas e uso indevido de uma série de conceitos relativos à MEE. Dentro desse contexto de ensino e produção de conhecimento em Psicologia e áreas afins é que este artigo se insere, pois tem o objetivo de apresentar e discutir sobre as concepções e aplicações da MEE na produção de conhecimento em Psicologia e áreas correlatas.

\section{Histórico e Conceituação}

A MEE é uma técnica de análise de dados que possui origem híbrida, em ao menos três diferentes ciências. De acordo com Kaplan (2000), a MEE foi influenciada pela psicometria do início do século XX, graças aos trabalhos seminais de alguns psicometristas (e.g. Pearson, Spearman, Thurstone, entre outros). O modelo da psicometria clássica estabeleceu o princípio de mensuração de características latentes humanas (e.g. habilidades cognitivas) por meio de tarefas comportamentais. Os psicólogos procuravam mensurar tais capacidades humanas por meio de uma série de tarefas (i.e. itens dos testes) e a identificação da estrutura dessas medidas era dada pelas intercorrelações entre esses itens. De acordo com Kaplan (2000), esse modelo de traço latente, que utilizava o modelo analítico da análise fatorial, foi a primeira influência para o desenvolvimento dos atuais modelos de equações estruturais. No decorrer do século XX vários outros psicometristas e estatísticos aperfeiçoaram o modelo da análise fatorial até o desenvolvimento do modelo atual da MEE.

Kaplan (2000) aponta que a outra influência é advinda dos modelos de equações múltiplas. Essa linha de tratamento de dados foi originalmente proposta por um geneticista chamado Wright, que estava interessado em compreender aspectos da morfologia animal a partir da análise simultânea de diferentes variáveis antecedentes. A partir do uso de equações de regressão múltipla, Wright criou a análise de trajetórias, em seus estudos seminais do início do século XX. Por outro lado, a lógica de análise de equações múltiplas também foi desenvolvida no seio da econometria. Em meados do século XX, os economistas estavam interessados em compreender fenômenos macroeconômicos por meio do emprego de múltiplas equações de regressão. De acordo com Kaplan (2000), essa questão é atual e a preocupação com a modelagem de equações múltiplas de dados longitudinais segue em discussão na econometria.

Dessa forma, a MEE tem uma origem histórica híbrida e aplicada a resolução de problemas práticos de testagem de modelos complexos, com múltiplas variáveis simultâneas e traços latentes. Por esse motivo, a MEE é apresentada por vários autores como uma mistura de análise fatorial com regressão múltipla e análise de trajetórias (e.g. Ullman, 2007). A MEE é uma técnica de equações estruturais ou equações múltiplas, mas se diferencia da análise de trajetórias 
porque trabalha com variáveis latentes, ou seja, medidas não diretamente observadas, mas sim derivadas de indicadores observáveis, como itens de uma escala de avaliação psicológica ou de comportamento organizacional. Dessa forma, a técnica utiliza princípios teóricos e lógicos dos modelos psicométricos da análise fatorial e a lógica de teste de equações múltiplas da econometria.

Como a maioria das técnicas de análise de dados, a MEE é útil para o teste de modelos teóricos em diferentes áreas do conhecimento. É utilizada, no contexto brasileiro, em pesquisas de Psicologia Organizacional e do Trabalho (e.g. Pilati \& Abbad, 2005), Psicologia Social (e.g. Gouveia, Martinez, Meira \& Milfont, 2001), Marketing (e.g. Frederico \& Robic, 2005), gestão de pessoas (e.g. Medeiros, Albuquerque \& Marques, 2005), entre outras áreas. Assim, demonstra-se como técnica útil para o desenvolvimento teórico de diversas subáreas da Psicologia, bem como para outras disciplinas que tenham a necessidade de mensuração e teste de relações entre fenômenos complexos e que exijam a análise de múltiplas relações simultâneas, entre as quais variáveis latentes.

Além disso, a MEE pode ser utilizada como procedimento de tratamento de dados em diferentes delineamentos de pesquisa em Psicologia e ciências humanas. Usualmente, a maioria das aplicações tem sido observada em pesquisas de delineamento correlacional, nas quais múltiplas variáveis são mensuradas e suas relações são testadas por meio da MEE, sem controle de variáveis. Mas a técnica também pode ser utilizada em delineamentos de pesquisa de outros tipos, como os quase-experimentais ou experimentos de campo ou os delineamentos experimentais clássicos (Bullock, Harlow \& Mulaik, 1994; Shadish \& cols., 2002). A aplicação de MEE nesse contexto traz várias vantagens para o pesquisador que está conduzindo experimentos, pois, de acordo com Shadish e cols. (2002),

\begin{abstract}
um simples experimento randômico nos aponta a ocorrência de um efeito. $O$ uso de modelagem causal nos ajuda a explicar o efeito por meio da modelagem de possíveis relacionamentos entre componentes moleculares da variável independente, de variáveis mediadoras, de variáveis moderadoras e da variável dependente (p. 404).
\end{abstract}

Em suma, verificados os pressupostos de análise, a MEE pode ser aplicada independentemente do delineamento de pesquisa, o que pode trazer luz para resultados de experimentos que exijam a avaliação de múltiplas variáveis, além do teste de relações diretas e indiretas simultâneas.

\section{MEE: Conceitos Básicos}

Segundo Bollen (1989), a MEE tem por lógica básica a reprodução da matriz de covariâncias populacionais por meio das covariâncias amostrais associadas às imposições de parâmetros determinadas pelo pesquisador. Se o modelo imposto for plausível, isso significa que covariâncias provenientes de dados amostrais podem reproduzir os dados populacionais associados ao conjunto de parâmetros do modelo estrutural. Modelos plausíveis indicam que a reprodução gerou resíduos reduzidos. Do contrário, se essa reprodução não foi plausível, os resíduos são elevados.
Com essa lógica, o modelo da MEE procura replicar um conjunto de dados observados por meio da imposição de parâmetros nas matrizes, que são as relações teóricas definidas pelo pesquisador. Essa característica é a principal diferença entre a MEE das outras técnicas de análise multivariada, pois a imposição dos parâmetros na matriz de relações entre as variáveis dá a ela um caráter confirmatório, pois exige do pesquisador uma pré-definição do tipo de relações existentes entre as variáveis do modelo em teste, que são operacionalizadas em termos de restrições nas matrizes. Por esse motivo, a MEE necessita que a) as medidas utilizadas pelo pesquisador sejam de boa qualidade psicométrica e b) modelos teóricos sólidos e fundamentados em pesquisas anteriores que permitam ao pesquisador estabelecer essas imposições (relações pré-definidas) com propriedade. Devido a essa última característica é que a MEE é entendida como uma técnica confirmatória, pois a modelação teórica sobre o que está sob investigação deve ter ocorrido antes da análise dos dados.

Uma outra questão de fundamental importância no campo e relacionada ao caráter confirmatório da técnica diz respeito à MEE estabelecer relações causais. Muitos autores têm discutido sobre a questão causal da MEE e o debate continua em aberto (e.g. Bullock \& cols., 1994; Hox \& Bechger, 1998). De acordo com Shadish e cols. (2002), relações causais dizem respeito a controles metodológicos e não estatísticos sobre variáveis de um estudo. Bullock e cols. (1994) argumentam que "se os dados são correlacionais por natureza, nenhum método estatístico pode alterar o delineamento" (p. 254).

Nessa mesma linha de argumentação, Hox e Bechger (1998) dizem que a maioria dos estudos que utilizam MEE é correlacional e que a interpretação causal do modelo final pode ser correta, mas que a MEE não consegue transformar "magicamente" dados de natureza correlacional em relações causais. Dessa forma, Hox e Bechger (1998) argumentam que os pesquisadores devem ter em mente que, se um modelo é corroborado pelos dados em estudos de MEE, isso não implica que esse modelo tenha sido provado como verdadeiro, mas sim que o modelo não foi falseado. Isso não implica na ausência de outros modelos concorrentes que possam, também, ser não-falseados. Esse princípio lógico argumentado pelos autores corrobora a noção epistemológica falsificacionista de produção de conhecimento (Popper, 1972), o que é totalmente coerente com as reais preocupações da ciência psicológica contemporânea. O que deve ser evitado é que os pesquisadores, por mal uso de conceitos de MEE, façam conclusões ou tomem decisões equivocadas, dado o princípio epistemológico subjacente à concepção da técnica.

Assim, a atribuição de causalidade entre dois fenômenos (i.e. A causa $\mathrm{B}$ ) está associada à capacidade do delineamento de pesquisa elaborado controlar a multiplicidade de fatores que podem, também, interferir na variação de B e isolar o efeito provocado por $\mathrm{A}$, chegando-se, então, à conclusão de que A causa B. A questão da "causa" em ciência está diretamente relacionada ao tipo de delineamento utilizado pelo pesquisador em sua investigação e não à metodologia estatística por ele utilizada para o controle matemático-estatístico dos dados empíricos. A noção de causa em MEE 
está estreitamente relacionada à lógica de imposição ou restrição de relações entre variáveis, algo realizado pelo pesquisador com suporte teórico e empírico precedente. Essa concepção também é relacionada à direção dos coeficientes de determinação, também estabelecidos pela perspectiva teórica do pesquisador. Mas esses aspectos não significam que a adequação das relações impostas indique relação causal entre variáveis, mas sim uma relação preditiva ou de determinação. Em suma, o problema da relação causal é um artefato metodológico (Shadish \& cols., 2002) e não matemático, como muito da discussão na literatura de MEE pode levar a entender.

Em MEE, uma das questões primordiais diz respeito à especificação do modelo. Isso é, o estabelecimento de relações entre as variáveis, a definição dos tipos de variáveis no modelo e a caracterização do tipo de modelo existente. Segundo Hox e Bechger (1998), o processo de especificação de um modelo é guiado pela combinação de elementos teóricos e de evidências empíricas de pesquisas anteriores. Uma característica comum em MEE, como forma de especificação, é a representação gráfica ou pictórica dos modelos estruturais. Essa representação possui uma simbologia de nomenclatura convencionada pelos pesquisadores do campo. As relações estruturais entre variáveis são representadas por diagramas. Nesses diagramas, as variáveis representadas por retângulos ou quadrados indicam variáveis observadas e variáveis representadas por círculos ou elipses indicam variáveis latentes. As variáveis latentes, por sua vez, estão associadas às variáveis observadas, pois os construtos latentes são aferidos, via de regra, por fenômenos observáveis do campo de investigação (e.g. os itens de um instrumento psicométrico de medida são os indicadores observáveis de construtos latentes que tais itens têm como função mensurar). Hair, Anderson, Tatham e Black (2005) definem variáveis latentes como a operacionalização de construtos em MEE. Construtos são variáveis não diretamente obervadas, mensuradas de forma indireta por meio de variáveis observadas. Por sua vez variáveis observadas ou manifestas são definidas por Hair e cols. (2005) como os indicadores de uma variável latente ou construto.

A Figura 1 é uma representação pictórica de um modelo de equações estruturais. Nessa figura, as variáveis latentes ou fatores são representados pela sigla ' $F$ ', as variáveis observadas pela letra "V" e os erros, associados a cada variável observada, pela letra "E". O distúrbio, erro associado a uma variável dependente, é representado pela letra "D”. Ressalta-se que variáveis latentes são aqui entendidas como fenômenos que não são mensurados via observação direta, mas sim de forma indireta por meio de indicadores observados. No caso da Psicologia é muito comum que estes indicadores observados sejam itens de instrumentos psicométricos, como defendido no âmbito da psicometria (Anastasi \& Urbina, 2000; Pasquali, 2003).

Como pode ser observado na Figura 1, as relações explicativas entre as variáveis são descritas por setas unidirecionais. Cada uma dessas setas pode ser descrita matematicamente como uma reta de regressão ${ }^{2}$. De acordo

2 Equação linear de regressão simples: $y=a+b x+e$; onde " $a$ " é o intercepto, o "b" é o coeficiente angular e o "e" o termo de erro. com Hox e Bechger (1998), essas relações também podem ser entendidas como cargas fatoriais, se relativas à análise fatorial confirmatória de instrumentos de medida. Já as setas curvas e bidirecionais representam correlação ou covariância entre variáveis. Um outro aspecto relevante que deve ser ressaltado em relação à Figura 1 é a predefinição do valor de uma relação de determinação ou carga fatorial entre a variável latente e uma variável observada (e.g. a relação entre "F1" e "V1" definida com valor um). Essa predefinição é importante para o estabelecimento da métrica em que as relações serão apresentadas na análise.

Um outro aspecto relevante na nomenclatura de MEE diz respeito ao tipo de variáveis dos modelos. Kaplan (2000) define que o primeiro é chamado de variáveis endógenas e se referem àquelas que recebem setas unidirecionais. Essas são conhecidas também como variáveis critério ou variáveis dependentes. Já as variáveis exógenas são aquelas que originam setas unidirecionais, sendo também conhecidas como variáveis preditoras ou variáveis independentes. Em MEE ocorrem casos nos quais uma mesma variável pode ser endógena ou exógena, ou seja, ela pode "receber" e "enviar" setas, comportando-se como variável critério e variável preditora simultaneamente. Isso é possível devido a aspectos teóricos de mediação entre variáveis, tipo de relação que pode ser testado em MEE.

Além da definição do tipo de variáveis para a especificação de um modelo, é crucial a definição da característica geral do mesmo. De acordo com Kline (1998), existem dois tipos básicos de modelos em MEE: recursivos e não-recursivos. Modelos recursivos têm como característica a ausência de covariância entre distúrbios de variáveis endógenas e a unidirecionalidade das relações de determinação entre as variáveis (i.e. não existem setas unidirecionais de retorno entre variáveis endógenas e exógenas). Modelos não-recursivos, por sua vez, possuem relação de dupla determinação entre variáveis (i.e. duas setas unidirecionais entre duas variáveis do modelo) e/ou correlações (i.e. setas bidirecionais) entre os distúrbios de variáveis endógenas.

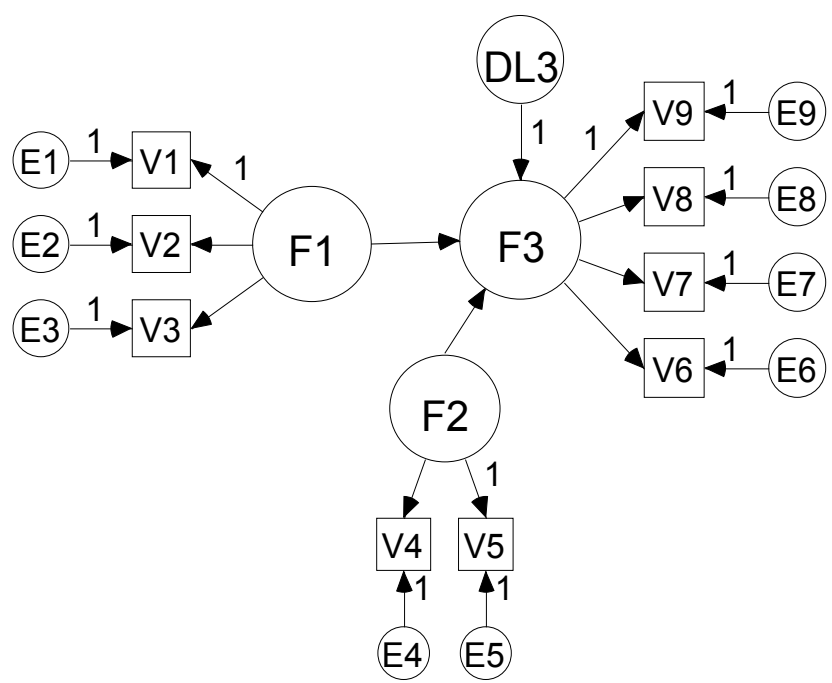

Figura 1. Exemplo de representação pictórica de modelo de equações estruturais. 
A Figura 1 é um exemplo prototípico de um modelo recursivo, pois as relações de determinação (setas) são todas unidirecionais, ou seja, partem dos fatores $(\mathrm{F})$ ou para outros Fs ou para variáveis observadas (Vs). Tomando-se como base o modelo da Figura 1, este poderia se tornar não-recursivo se, por exemplo, uma nova seta unidirecional fosse inserida no modelo, indicando uma relação de "retorno" entre F3 e F2. A característica de um modelo de MEE é crucial para o processo de identificação de modelos estruturais, pois modelos não-recursivos têm um número maior de parâmetros para serem estimados, o que traz certas exigências para o processo de análise.

Em relação à especificação de modelos estruturais, também é necessária a definição dos dois sub-modelos existentes. $\mathrm{O}$ primeiro refere-se ao modelo de mensuração, que se traduz na relação entre variáveis latentes e as variáveis observadas (i.e. mensuradas na realidade empírica), que permitem a mensuração dos fenômenos latentes existentes no modelo. Na Figura 1, o modelo de mensuração é representado pela relação entre os fatores (F) e as variáveis observadas (Vs). A adequação e ajuste do modelo de mensuração é crucial para que um modelo de equações estruturais possa ser testado. Esse processo de aferição do modelo de mensuração é conhecido como análise fatorial confirmatória (AFC). O segundo sub-modelo existente é o chamado modelo estrutural ou diagrama estrutural, que trata das relações entre variáveis latentes do modelo em teste. Na Figura 1, o modelo estrutural é representado pelas relações entre os fatores (Fs).

Outro aspecto para o teste de modelos estruturais é a identificação do modelo. Pelo seu caráter confirmatório, a MEE impõe relações entre variáveis que são simultaneamente expressas por uma matriz de dados. Por essas relações não serem informações presentes na matriz de covariâncias é necessário que as mesmas sejam estimadas por métodos matemáticos. Essa estimação tem como ponto de partida os dados presentes na matriz. A referida característica gera um problema de falta de informações para o processo de estimação. Quanto maior for o número de parâmetros (relações entre variáveis, variâncias e covariâncias a serem estimadas), maior a dificuldade para a estimação. De acordo com Kaplan (2000), a identificação de um modelo estrutural ocorre quando o número total de parâmetros a serem estimados pode ser unicamente representado pelo conjunto de dados da matriz de covariâncias amostrais. A regra mais simples e difundida para se estabelecer o índice de identificação do modelo de equações estruturais é a regra da contagem (Byrne, 2001; Kaplan, 2000; Ullman, 2007). Na matriz de covariâncias ou correlações o número total de elementos não redundantes é igual a $1 / 2 p(p+1)$ onde " $\mathrm{p}$ " é o número total de pontos da matriz de covariância ou correlações.

Se o número total de parâmetros for igual ao número de elementos não redundantes, tem-se um modelo identificado, ou seja, os graus de liberdade (GL) ${ }^{3}$ desse modelo são iguais a zero. Se for menor ou maior ao número, tem-se, respectivamente, modelos sub-identificados e superidentificados. Para que seja possível a estimação dos parâmetros, é essencial que

3 Os graus de liberdade de um modelo de equações estruturais são definidos pela diferença entre o número de elementos não redundantes na matriz de correlações e o número de parâmetros do modelo. o modelo em teste seja ao menos superidentificado, ou seja, que tenha GL maior que zero.

\section{Pressupostos da MEE}

Como toda técnica pertencente ao modelo linear geral (MLG), a MEE exige que os dados possuam certos pressupostos para serem submetidos a modelagem. Maiores discussões sobre essa questão podem ser encontradas em Tabachnik e Fidell (2007). Textos específicos dedicam-se a desenvolver argumentações sobre a importância da análise exploratória de dados para adequação ao modelo linear geral, explanando sobre técnicas de identificação e resolução de problemas de ausência de pressupostos para análise de dados. Para tanto, sugere-se ao leitor que busque essa literatura específica, pois no presente trabalho pretende-se abordar apenas algumas das questões relativas aos pressupostos dos dados estatísticos, aquelas consideradas mais relevantes na MEE.

Um dos mais importantes pressupostos dos dados para a MEE é a normalidade das distribuições das variáveis. Há necessidade de que os dados em análise respeitem pressupostos de normalidade univariada e multivariada. A MEE utiliza diferentes métodos de estimação para o cálculo dos parâmetros do modelo. $\mathrm{O}$ mais difundido dos métodos é o da máxima verossimilhança (MLE), que exige que os dados tenham normalidade para a estimação. Nesse método, além da normalidade univariada, é necessário que os dados possuam normalidade multivariada. De acordo com Kline (1998), a normalidade multivariada significa que a) os dados tenham todos distribuição univariada normal; b) a distribuição conjunta de qualquer combinação de variáveis deve ser normal e c) todos os gráficos de dispersão bivariados devem ser lineares e homocedásticos. Segundo Kline (1998), de forma a permitir a análise conjunta da normalidade das variáveis, programas de modelos de equações estrurais, como o AMOS, calculam índices de assimetria e achatamento multivariados, por meio do coeficiente de Mardia (1971). Dessa forma, é possível que o pesquisador avalie essa característica da distribuição de dados e utilize um método de estimação de parâmetros coerente com o padrão de normalidade das variáveis.

Existem métodos de estimação dos parâmetros que não exigem o pressuposto da normalidade dos dados, como o ADF (Método Assintótico Isento de Pressupostos de Distribuição). O MLE, entretanto, apresenta algumas vantagens relevantes, como a facilidade do processamento estatístico e tamanho reduzido da amostra, além da possibilidade de estabelecimento de diversos índices de adequação. O pesquisador deve estar atento a esses aspectos para poder estimar com validade e precisão os parâmetros do modelo. Caso o pressuposto da normalidade não seja atingido, mesmo após a aplicação de procedimentos de transformação das variáveis (e.g. Osborne, 2002), o pesquisador pode optar por utilizar outros métodos de estimação, como o ADF. De acordo com Kline (1998), além dos processos de transformação de variáveis, o pesquisador também pode optar pelo tratamento dos casos extremos como forma de incrementar a normalidade dos dados.

Outro pressuposto de grande importância é relativo aos casos omissos, ou dados faltosos. De acordo com Kline (1998), esse é um problema comum em pesquisa de diferentes áreas, mas que deve ser tratado para não influenciar o processo de 
análise por meio da MEE. A falta de dados brutos ocasiona problemas na estimação dos parâmetros de um modelo estrutural, pois os coeficientes da matriz de covariância são obtidos com números não homogêneos de dados brutos. De acordo com Little e Rubin (1987), esse tipo de ocorrência deve ser superado pelo uso de técnicas de estudo do caráter de sistematicidade de dados omissos e pela posterior estimação dos valores omissos por meio do uso de um procedimento adequado de estimação. Essa estimação ou imputação de dados brutos é essencial para que as covariâncias ou correlações da matriz sejam calculadas com um número homogêneo de informações. Portanto, a questão de dados omissos é crucial em análises do modelo linear geral e várias estratégias de resolução dessas questões estão disponíveis na literatura especializada.

Kline (1998) ainda discute que, além dos pressupostos até aqui comentados, o pesquisador também deve estar atento para questões de homocedasticidade, heterocedasticidade, singularidade e valores ou casos extremos. Apesar de magnitude e influência menor sobre o processo de MEE, esses pressupostos também devem ser observados para que não ocorram problemas com o processo de estimação dos parâmetros e índices associados à MEE. Como salientado no início dessa seção, não é função do presente trabalho esgotar a discussão sobre pressupostos de análise, mas apenas alertar aos leitores sobre aspectos dos pressupostos considerados como mais relevantes.

\section{Informações Essenciais em Relatos de MEE}

Devido às suas características peculiares como técnica de análise e tratamento de dados, o uso da MEE deve ser relatado levando-se em consideração alguns aspectos. Tais aspectos são relativos à clareza de transmissão das informações e ao tipo de informações que devem ser transmitidas para que a comunidade científica possa ter acesso aos aspectos cruciais que levaram o pesquisador às decisões tomadas e conclusões alcançadas. Na literatura internacional, são observados artigos científicos que buscam discutir esses aspectos do relato de estudos que utilizam MEE (e.g. Boomsma, 2000; McDonald \& Ho, 2002). Como o objetivo do presente trabalho é apresentar elementos gerais e básicos para o uso criterioso da MEE no contexto da pesquisa brasileira, decidiu-se por apresentar alguns dos princípios de apresentação de resultados discutidos na literatura internacional da área. Espera-se que essa discussão possibilite o aprimoramento do tema na pesquisa em Psicologia e em áreas correlatas no Brasil.

Os cuidados no relato de pesquisas com uso de MEE devem seguir aspectos relativos aos conceitos básicos de MEE. Portanto, pode-se observar que alguns dos aspectos aqui tratados são conceituados em seções anteriores. Por um motivo de exposição lógica dos princípios do relato da MEE, os elementos cruciais serão apresentados na seqüência lógica de preocupação do pesquisador. Assim, os relatos de MEE devem considerar os seguintes elementos: a) aspectos teóricos; b) elaboração de modelos (relações hipotetizadas entre as variáveis); c) determinação teórica e descrição dos aspectos psicométricos das medidas do modelo; d) especificação do modelo (de suas duas partes, a saber, mensuração e estrutural); e) identificação do modelo (i.e. relação parâmetros versus pontos de dados da matriz de correlações, representa- ção gráfica do modelo hipotético, fixação da escala de medida do modelo de mensuração); f) descrever métodos de estimação de parâmetros; g) definir índices de ajuste e valores de referência para cada um; h) apresentar coeficientes estimados e seu teste de significância; i) apresentar índices de ajuste do modelo; j) relatar resíduos; k) descrever possíveis índices de modificação do modelo; e l) justificar teoricamente possíveis modificações do modelo hipotetizado.

O primeiro passo para se estabelecer um relato de MEE com os devidos cuidados está relacionado aos aspectos teóricos do estudo. Como já discutido anteriormente e partilhado por diferentes autores do campo (e.g. Bullock \& cols., 1994; Byrne, 2001; Kaplan, 2000; Ullman, 2007), a MEE possui um caráter confirmatório. Por esse motivo, essa não é uma técnica adequada para explorar relações entre variáveis, o que pode ser efetuado por meio de outras técnicas de estatística multivariada (para maiores detalhes veja Tabachnik \& Fidell, 2007 ou Hair \& cols., 2005). Por essa característica, a elaboração teórica de modelos hipotéticos é tão importante no contexto da MEE, pois as relações que serão construídas pelo pesquisador entre as variáveis devem ser embasadas em pressupostos teóricos e evidências empíricas anteriores. Essa característica é essencial para que o pesquisador possa alcançar resultados teoricamente coerentes e modelos ajustados aos dados. Caso o campo de investigação não possua tais evidências, recomenda-se que o pesquisador inicie os estudos com a utilização de outras técnicas multivariadas de análise de dados que não possuam característica confirmatória.

O levantamento e a descrição de aspectos teóricos permitem ao pesquisador o desenho de modelos a serem investigados por meio da MEE. Essa etapa do processo refere-se à formulação de hipóteses de relações entre variáveis. Essa formulação de hipóteses permitirá ao pesquisador definir as relações de determinação entre variáveis do modelo estrutural. Dentro de uma perspectiva teórica podem existir modelos concorrentes e o pesquisador deverá modelar teoricamente seu conjunto de hipóteses da forma mais coerente e lógica possível, para que suas hipóteses façam sentido no processo de construção de conhecimento em um campo do saber. A escolha e a definição das relações mais procedentes entre variáveis são relativas à modelagem teórica de uma área do saber e dizem respeito a um aspecto essencial para a especificação e identificação de um modelo estrutural a ser testado por meio da MEE.

Outro aspecto de fundamental importância e parte integral de um relato de MEE é relativo à fundamentação teórica das medidas no modelo. A tradição teórica da MEE está centrada nos modelos de traço latente, presentes na Psicologia desde o princípio do século XX (Anastasi \& Urbina, 2000; Kaplan, 2000; Ullman, 2007). A teoria psicométrica pressupõe que a resposta a um conjunto de itens de um instrumento esteja associada a um traço latente (i.e. habilidade, atitude, etc) presente nos esquemas cognitivos dos indivíduos. A partir desse pressuposto, modelos teóricos foram desenvolvidos para a construção de medidas de fenômenos latentes. Os modelos da psicometria clássica ou da psicometria moderna, respectivamente Teoria Clássica dos Testes (TCT) e Teoria de Resposta ao Item (TRI), definem conceitos e procedimentos para a estruturação teórica e validação empírica de medidas (Pasquali, 2003). O pesquisador que faz uso da MEE deve ter em mente que os instrumentos de medida devem ser válidos 
e fidedignos. Assim, é fundamental que as medidas utilizadas em um estudo de MEE tenham sido desenvolvidas a partir de estruturas teóricas sólidas e que já tenham sua validade demonstrada, preferencialmente em estudos anteriores. Além disso, é fundamental que o pesquisador também relate as características psicométricas das medidas utilizadas, dando possibilidade ao leitor de julgar a qualidade psicométrica dos instrumentos de medida.

O quarto aspecto a ser considerado pelo pesquisador em um relato de MEE diz respeito à especificação do modelo. Esta necessita da explicitação de dois aspectos cruciais. O primeiro é relativo à especificação do modelo de mensuração, que diz respeito à qualidade das medidas utilizadas para aferir as variáveis latentes. Para tanto, o pesquisador deve submeter as medidas à análise fatorial confirmatória (AFC) por meio da MEE e testar a qualidade das medidas a partir dos índices de adequação do modelo de mensuração, entre outros indicadores. Essa etapa é crucial para que problemas de estimação do modelo estrutural não sejam atribuídos a deficiências das medidas utilizadas pelo pesquisador. $\mathrm{O}$ segundo é relacionado ao modelo estrutural e define-se como as relações de determinação e covariâncias que as variáveis exógenas e endógenas terão entre si. A definição do modelo estrutural implica na atribuição de restrições na matriz de dados, que são os parâmetros que o processo de análise de dados deve estimar. Como a MEE trabalha intensamente com modelos gráficos, essa especificação do modelo estrutural pode ser representada por meio dos diagramas de MEE, como aquele apresentado na Figura 1 do presente trabalho.

$\mathrm{O}$ processo de especificação do modelo traz à tona a necessidade da identificação do modelo que será testado. $\mathrm{O}$ pesquisador que está a relatar um estudo com uso de MEE deve explicitar em sua argumentação as características de identificação do modelo, ou seja, a relação entre parâmetros fixos versus parâmetros livres do mesmo. Para que o modelo em teste seja passível de estimação este deve ter uma relação adequada entre parâmetros livres (a serem estimados) e parâmetros fixos, ou seja, o grau de liberdade deve ser superior a zero. Essa informação já foi apresentada anteriormente por meio da regra básica de identificação do modelo. O pesquisador deve relatar essa relação de forma a propiciar ao seu leitor esta informação. Além disso, também faz parte da identificação do modelo a definição da escala de trabalho do modelo de mensuração que, como já comentado anteriormente, é feita por meio da fixação, geralmente no valor um, de uma das cargas fatoriais de uma variável latente do modelo.

Uma outra informação que os pesquisadores devem relatar é o método de estimação. A questão do método de estimação é essencial para que o leitor possa compreender a qualidade dos dados estimados, assim como as ameaças ao processo de estimação, dadas as características dos dados. Segundo Foguet e Gallart (2001), são frequentemente observados na literatura especializada quatro métodos de estimação de parâmetros em MEE: a) Quadrados Mínimos Não-Ponderados (ULS); b) Quadrados Mínimos Ponderados (GLS), que possuem a normalidade como pressuposto de trabalho; c) Máxima Verossimilhança (MLE), que também exige normalidade dos dados; e d) Método Assintótico Isento de Pressupostos de Distribuição (ADF) que não exige pressupos- to de normalidade das variáveis. Os autores comentam que os métodos mais frequentemente utilizado são o MLE e o GLS, provavelmente devido à sua maior difusão nos estudos seminais de MEE. Ainda de acordo com Foguet e Gallart (2001), a aparente superioridade do $\mathrm{ADF}$, devido a não necessidade de que os dados atinjam o pressuposto de normalidade, não é real, pois esse método exige grandes contingentes amostrais para proporcionar estimações confiáveis. Em relação ao tamanho da amostra, o MLE e o GLS funcionam bem entre 200 e 500 indivíduos, sendo possível que sejam adequados também para amostras maiores a depender das características dos dados em análise. Por questões como essa, é importante que o pesquisador discuta e selecione métodos de estimação adequados às características amostrais de seu estudo.

Após a definição dos aspectos relativos à estimação, o pesquisador deve posicionar-se sobre os índices de ajuste que serão utilizados para teste de adequação do modelo. Esse aspecto da MEE é importante e o desenvolvimento de várias estratégias de teste de adequação possibilitou o surgimento de vários e diferentes métodos para a estimação do ajuste de um modelo estrutural aos dados, tendo em vista que essa é uma das informações mais relevantes em MEE. Segundo Hox e Bechger (1998), o índice clássico de ajuste de um modelo é o qui-quadrado $\left(\chi^{2}\right)$. Para esses autores o grande problema desse índice é seu alto grau de sensibilidade ao tamanho das amostras, o que torna o índice significativo, o que não é desejado pois indica a rejeição do modelo. O contrário também é verdadeiro, pois se a amostra é reduzida, o índice será não-significativo, o que pode levar o pesquisador a aceitar um modelo quando outros concorrentes são mais verossímeis. Para se lidar com situações como essa, a área de MEE desenvolveu grande quantidade de índices de adequação de modelos de equações estruturais.

Ullman (2007) classifica os índices de adequação em cinco categorias. A primeira é denominada índices comparativos e trabalha com a lógica de comparação do $\chi^{2}$ de modelos independentes com o $\chi^{2}$ do modelo em teste. São exemplos desses índices o NFI (Normed Fit Index), o NNFI (Non-Normed Fit Index), o IFI (Incremental Fit Index) e o CFI (Comparative Fit Index). Um índice peculiar nessa categoria, segundo a classificação da autora, é o RMSEA (Root Mean Square Error of Approximation) que testa o ajuste próximo do modelo por meio da comparação entre o modelo em teste e um modelo saturado com o mesmo conjunto de dados. A literatura aponta que valores entre 0,90 e 0,95 são indicadores de ajuste suficiente e valores acima de 0,95 são considerados bons ajustes (Ullman, 2007). Em relação ao RMSEA, espera-se um valor menor do que 0,08 (Hancock \& Freeman, 2001; MacCallum, Browne \& Sugawara, 1996) considerado como adequado, dado o poder do teste. Uma outra característica relevante do RMSEA é a possibilidade de estimação de intervalos de confiança do índice, o que auxilia no julgamento de ajuste próximo. Esses valores de intervalo de confiança do RMSEA devem ser relatados pelo pesquisador. A segunda categoria, índice de adequação absoluto, tem apenas um representante, o MFI. Esse realiza o teste de adequação por meio da relação entre o $\chi^{2}$ do modelo e seus graus de liberdade. Este índice é considerado absoluto porque não compara o modelo em teste com qualquer outro possível modelo. 
A terceira categoria de índices são aqueles relativos à proporção de variância explicada. São exemplos dessa categoria de índices: o GFI (Goodness of Fit Index) e o AGFI (Adjusted Goodness of Fit Index). Esses índices baseiam-se no cálculo da proporção da variância explicada por meio da estimação. No caso destes, também se espera valores próximos a 1,0 para a indicação de ajuste do modelo. De acordo com Hox e Bechger (1998), pode-se aceitar um modelo com valores de 0,90 e um valor considerado indicador de um bom ajuste seria o de 0,95 .

A quarta categoria de índices diz respeito ao grau de parcimônia do modelo testado. Fazem parte dessa categoria o PGFI (Parcimony Goodness of Fit Index), o AIC (Akaike Information Criterion) e o CAIC (Consistent Akaike Information Criterion). Esses índices dão informações da adequação do modelo por meio de uma relação entre o número de parâmetros estimados e o número de pontos de dados na matriz de covariâncias. De acordo com Byrne (2001), o AIC e o CAIC permitem a comparação de dois ou mais modelos. Aqueles modelos que obtiverem valores menores indicam que possuem melhor ajuste. Para a análise dos índices é importante considerar os graus de liberdade do modelo, que nesse caso está relacionado ao número de parâmetros estimados. No teste de adequação de modelos estruturais é possível a comparação de modelos concorrentes por meio da restrição de um número diferente de relações entre variáveis, o que diferencia o número de parâmetros a serem estimados. Se o pesquisador utiliza o AIC ou o CAIC para teste de adequação, ele deve considerar, além da diminuição do valor absoluto dos índices, o valor da razão crítica do novo modelo e da diferença dos indicadores para todos os modelos em teste. Então, o índice de melhora de ajuste se dá pela razão crítica encontrada, que é a relação entre o valor do índice e o número de parâmetros, e não simplesmente pela diminuição do valor absoluto dos indicadores.

A última categoria de índices descrita por Ullman (2007) é a dos índices de ajuste baseados em resíduos. O principal representante é o RMR (Root Mean Square Residual) e sua variação SRMR (Standardized Root Mean Square Residual). Ambos avaliam a "distância" entre os elementos preditos por meio da estimação dos parâmetros do modelo e os dados observados na matriz de covariâncias. Nesse último caso, esperam-se valores próximos de zero como indicadores de ajuste do modelo. O pesquisador que desenvolve um relato de MEE deve indicar ao seu leitor quais índices serão utilizados para aferir o grau de ajuste do modelo, bem como, quais os valores de referência encontrados no processo de modelagem dos dados. Todos os valores dos índices de ajuste apresentados no presente artigo funcionam como regras de tomadas de decisão, de forma que cabe ao autor do relato que faz uso de MEE, a seleção dos índices e a definição dos critérios de corte com base em argumentação consistente sobre os índices de adequação.

Após a descrição desses elementos preliminares, os autores devem procurar apresentar um conjunto sistemático de informações que permita ao leitor avaliar os resultados alcançados. Para tanto, são essenciais os seguintes elementos: apresentação dos coeficientes (determinação e covariância) e seus respectivos graus de significância; apresentação dos índices de ajuste do modelo e apresentação dos resíduos do modelo testado. É fundamental para o leitor de um relato de MEE que ele tenha acesso completo às informações padronizadas dos coeficientes que indicam as relações entre as variáveis do modelo. Isso porque essa informação possibilita a avaliação do grau e magnitude das relações entre variáveis além do seu nível de significância, reforçando a dimensão do teste das hipóteses específicas de um modelo, permitindo assim melhor fundamentação para a discussão e desenvolvimento teórico dos resultados do estudo. Outro elemento que deve estar presente no relato são índices de adequação com os respectivos valores obtidos. Esses indicadores permitirão ao leitor acessar a qualidade do modelo como um todo. Juntamente com os índices de adequação, é importante o pesquisador apresentar informações sobre os resíduos da análise. Essa informação complementar pode ser apresentada de forma sintética ou por meio da matriz de resíduos e serve para o leitor complementar o julgamento sobre a adequação do modelo e das imposições teóricas sobre os dados.

Análises por meio de MEE podem indicar possíveis alterações nas relações entre variáveis que permitem viabilizar a melhora do ajuste do modelo aos dados. Essas indicações são fornecidas pelos índices de modificação e devem ser cuidadosamente trabalhadas em um relato científico. Uma dimensão dessa questão diz respeito aos índices de modificação propriamente ditos. De acordo com Ullman (2007), existem três tipos básicos de índices de modificação: Lagrange Multiplier (LM), Wald Test e a comparação de $\chi^{2}$ de modelos alterados. A comparação de $\chi^{2}$ é uma estratégia interessante e funcional em modelos que têm característica aninhada (Foguet \& Gallart, 2001). Já o LM permite o teste e comparação do incremento de ajuste a partir da inserção de parâmetros específicos no modelo e, portanto, exige a comparação desses elementos a partir do teste de modelos específicos. De acordo com Ullman (2007), o Wald Test trabalha em uma perspectiva parecida do LM, mas estima a alteração do ajuste por meio da eliminação de parâmetros do modelo.

A partir do uso de índices de modificação é possível para o pesquisador alterar um modelo estrutural inicial, mas o processo de alteração, graças ao caráter confirmatório da MEE, não pode ser unicamente baseado nos indicadores estatísticos de modificação. Para tanto, é fundamental que o pesquisador tenha fundamentação teórica para modificação do seu modelo inicial. Foguet e Gallart (2001) apresentam alguns elementos que são esclarecedores para a modificação de modelos estruturais. Segundo os autores, o processo de modificação das relações estruturais de um modelo em MEE deve buscar consistência teórica. Assim, o pesquisador não pode proceder a modificações extensas no modelo e reavaliar seu grau de ajuste. Isso porque o processo de modificação está relacionado ao problema da capitalização da chance, em que as alterações podem evocar erros de tipo I e de tipo II no modelo. Esses autores argumentam que é válido o processo de modificação em pesquisas de MEE, mas que essas alterações devem ser feitas com base em elementos teóricos relevantes e não exclusivamente nos índices de modificação. Portanto, o pesquisador desenvolvendo um relato de MEE deve preocupar-se com os indicadores de modificação do modelo, mas procurar todas as evidências teóricas para efetivar tais modificações. 
Devido às suas peculiaridades, o relato de um trabalho de pesquisa que tenha utilizado a MEE como estratégia de análise de dados e teste de modelos devem considerar os aspectos aqui relatados. Isso porque tais aspectos permitem ao leitor de resultados de MEE possuir informações suficientes para julgar e contrapor os resultados e conclusões relatadas pelo autor. Além disso, tais cuidados também auxiliam ao próprio pesquisador a não chegar a julgamentos errôneos ou falácias devido à falta de cuidado com vários dos aspectos relativos à MEE. Como já foi salientado anteriormente, há várias questões teórico-metodológicas relativas ao uso da MEE que não podem ser esquecidas pelo pesquisador, justamente para permitir que o desenvolvimento teórico de um campo não seja limitado devido ao uso inadequado de estratégias de teste estrutural de modelos teóricos. Como forma de estabelecer um princípio guia para o pesquisador que faz uso da MEE, a Figura 2 apresenta o conjunto de aspectos que devem ser considerados no relato de pesquisa de MEE, como forma de síntese do que foi abordado nessa seção.

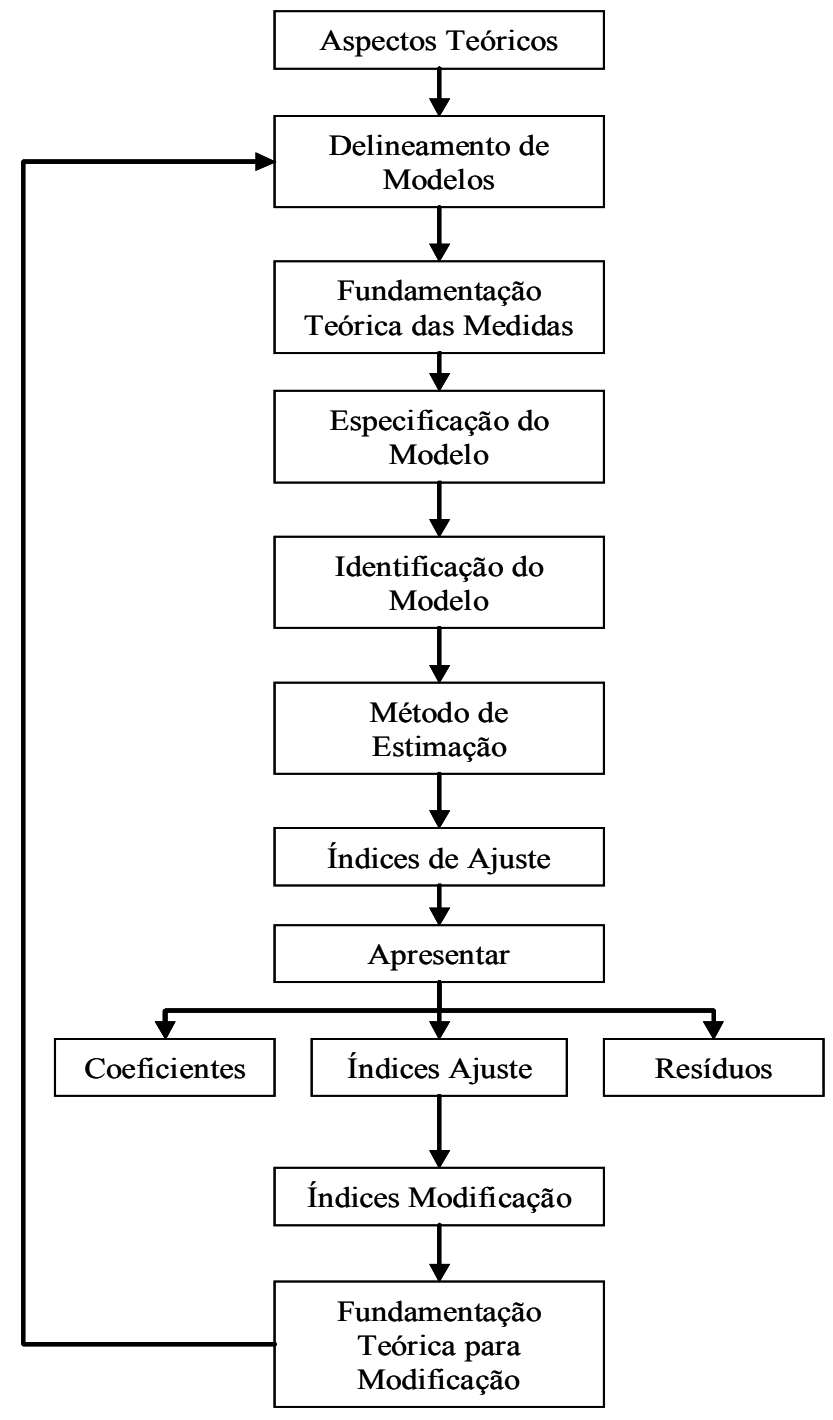

Figura 2. Aspectos a serem considerados em um relato MEE.

\section{Exemplificação de Resultados de uma AFC}

Como forma de elucidar alguns dos aspectos comentados no artigo, principalmente os concernentes à apresentação dos resultados de MEE (parte inferior da Figura 2), apresenta-se a seguir um exemplo de análise fatorial confirmatória (AFC) descrito por Arbuckle e Worthke (1999) no manual do usuário do AMOS 4.0. É importante ressaltar ao leitor que existem vários programas estatísticos que realizam análise de MEE. Para aqueles interessados, recomenda-se a leitura de Ullman (2007) que apresenta uma síntese comparativa entre tais programas computacionais.

Recorda-se que a AFC é uma possível aplicação da MEE. Optou-se por apresentar uma AFC porque é um tipo de análise frequentemente utilizado por pesquisadores em Psicologia e áreas correlatas, como forma de aferir a validade de construto de medidas.

A Figura 3 apresenta o modelo estrutural do exemplo de análise confirmatória. Deve-se observar que há valores prédefinidos, uma carga fatorial para cada fator, que definem a métrica da análise. Esses valores referem-se àqueles, sem erro padrão, apresentados na Tabela 1.

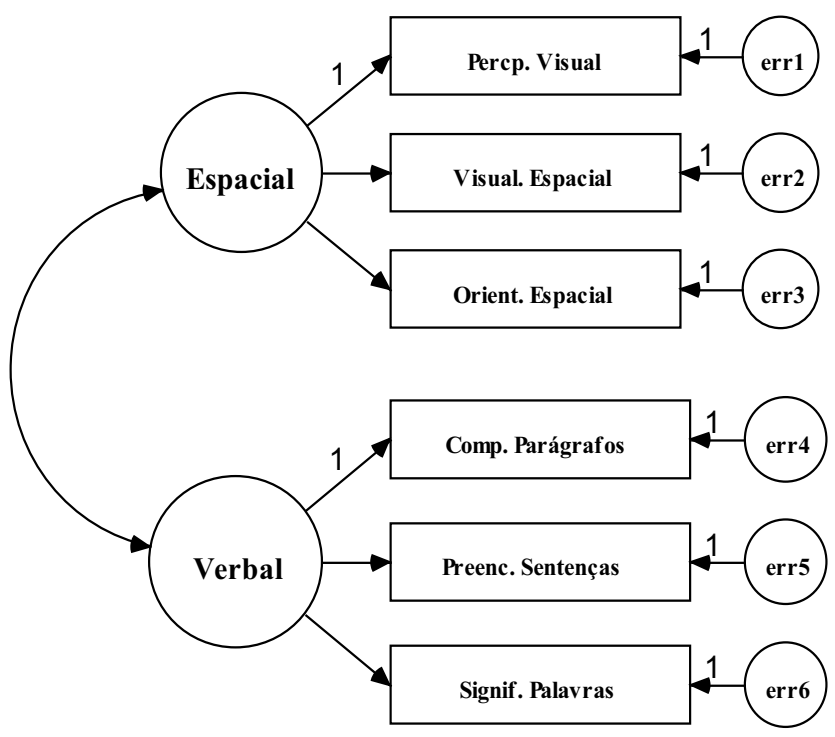

Figura 3. Diagrama da AFC exemplificada por Arbuckle e Worthke (1999).

A Tabela 1 apresenta os resultados da análise fatorial confirmatória. As informações desta tabela são as básicas que devem ser relatadas em qualquer pesquisa de MEE.

Como pode ser observado na Tabela 1, são apresentadas informações de cargas fatoriais, padronizadas e não-padronizadas, erro padrão de cada coeficiente (valor entre parênteses ao lado da carga não-padronizada) e coeficientes de correlação múltipla de um instrumento para aferição de habilidades espaciais e verbais. Esse instrumento é hipotetizado com dois fatores latentes (i.e. espacial e verbal) que possuem três indicadores ou variáveis observadas associados a cada um: percepção visual, visualização espacial e orientação espacial para o fator espacial; e compreensão de parágrafos, preenchimento de sentenças e significado das palavras para o fator verbal. 
Tabela 1. Coeficientes de determinação (cargas fatoriais), erro padrão e correlação múltipla ao quadrado (SMC) da AFC descrita por Arbuckle e Worthke (1999).

\begin{tabular}{lcccc}
\hline & \multicolumn{2}{c}{ Cargas fatoriais não-padronizadas } & Cargas fatoriais padronizadas & SMC \\
\cline { 2 - 5 } & Espacial & Verbal & Espacial & Verbal \\
\cline { 2 - 5 } Percepção visual & 1,00 & 0,70 & 0,49 \\
Visualização espacial & $0,61(0,14)$ & 0,65 & 0,43 \\
Orientação espacial & $1,20(0,27)$ & 0,74 & 0,84 & 0,77 \\
Compreensão de parágrafos & & & & 0,83 \\
Preenchimento de sentenças & & 1,00 & & 0,68 \\
Significado das palavras & & $1,33(0,16)$ & & 0,84 \\
\hline
\end{tabular}

Os índices de significância de cada carga fatorial dessa análise são determinados por meio da relação do valor da carga fatorial pelo seu erro padrão. Essa divisão dá a informação da razão crítica (RC), elemento crucial no relato de resultados de MEE. Usualmente valores maiores do que 1,96 e menores do que - 1,96 são considerados significativos em um teste bicaudal. No caso desse exemplo, todos os coeficientes estimados obtiveram valores significativos, indicando que existe menos de $5 \%$ de probabilidade que os coeficientes, na verdade, sejam iguais à zero. Aqueles que possuem valor 1 (um) associados são os que foram pré-fixados, com o intuito de determinação da métrica de apresentação dos coeficientes e por isso não possuem erro padrão associado.

Com relação aos índices de ajuste, essa análise obteve GFI $=0,97$ e CFI $=1,0$, o que indica altíssimo grau de ajuste para os dois tipos de índices. Já o RMSEA é igual a 0,00, com intervalo de confiança de $90 \%$ variando de 0,00 a 0,137 . Essa informação também indica ajuste próximo desse modelo de análise fatorial, apesar de uma margem alta de variação no intervalo de confiança. Sobre os resíduos, observa-se a existência de alguns valores acentuados, como pode ser observado pelo valor do RMR, que é igual a 1,68, o que indica que há elementos a serem aprimorados no presente modelo de AFC. Não são observados índices de modificação na presente análise.

\section{Limitações no Uso da MEE}

A MEE é uma técnica que traz várias possibilidades para o desenvolvimento teórico e metodológico em um campo do saber, como a Psicologia. Mas como qualquer técnica, ela não é capaz de abarcar a especificidade de todo e qualquer tipo de fenômeno e, portanto, possui limitações em seu uso.

A primeira questão que deve ser pontuada é que a MEE e seus modelos mais difundidos fazem parte da família de técnicas multivariadas pertencentes ao modelo linear geral. Desta feita, ela não é adequada e nem capaz de avaliar relações entre variáveis que não possuam um padrão linear de relacionamento. Nesse caso, o pesquisador deve lançar mão de alternativas que possam lidar com esse tipo de situação. A evolução natural dos modelos matemáticos da MEE deve passar pelo desenvolvimento de técnicas e procedimentos não-lineares, visando dar resposta para esse tipo de situação. Há notícias de que alguns programas específicos de MEE estão implementando resoluções de análise desse tipo. Uma alternativa para se lidar com essa situação seria trabalhar com matrizes de correlações ou covariâncias, base da MEE, adequadas para diferentes tipos de dados. Sugere-se que o leitor interessado busque informações específicas na literatura especializada.

Uma outra limitação do uso da MEE é o tamanho das amostras que devem ser utilizadas para que os dados sejam submetidos à MEE. Isso porque os estimadores da MEE exigem variabilidade acentuada para realizar o processo de estimação. No caso dos estimadores que pressupõe normalidade multivariada dos dados, o tamanho das amostras é menor se comparado àqueles que são livres de distribuição, mas mesmo assim ainda são amostras grandes. Essa característica pode "afugentar" os pesquisadores que trabalham com delineamentos de menor número de sujeitos, como os delineamentos experimentais. Mas mesmo assim existem possibilidades de aplicação de procedimentos de equações múltiplas e análise de trajetórias que poderiam ser úteis nesses casos. Vale ressaltar que a discussão sobre esse tópico ainda está aberta na literatura especializada, o que indica que há situações em que, dadas algumas características amostrais, é possível fazer uso da MEE sem amostras de número elevado de componentes. O que é relevante nesse contexto é a tentativa do pesquisador em buscar soluções para as limitações da técnica para que seu uso possa ser aplicado na busca de avanços nas diversas áreas do saber.

\section{Conclusões e Perspectivas Futuras da MEE}

A MEE traz grandes e importantes possibilidades para o desenvolvimento teórico da Psicologia e de outras áreas do conhecimento. Atualmente, o debate no campo da MEE segue em ritmo acelerado, com o desenvolvimento de novos modelos de análise adequados para a resolução de questões empíricas recorrentes em diferentes campos do conhecimento.

Uma dessas aplicações está relacionada aos fenômenos multinível. A teoria multinível de compreensão de fenômenos e modelagem de dados está interessada no desenvolvimento de estratégias para a produção de conhecimento em diferentes campos, como a teoria de comportamento organizacional (e.g. Kozlowski \& Klein, 2000) e a avaliação educacional (e.g. Willms, 1999). Essa perspectiva de modelagem teórica e de processo de análise de dados já está presente há certo tempo nas discussões da literatura de MEE. Kaplan (2000) 
apresenta o modelo geral da MEE multinível, com a aplicação dos devidos elementos de modelos hierárquicos aninhados para a matemática linear da MEE. Esse desenvolvimento do modelo da MEE é de extrema relevância para o avanço no campo, de forma a possibilitar o teste de modelos multinível de fenômenos em diferentes contextos, permitindo uma modelagem teórico-empírica que considere a complexidade de muitos fenômenos humanos e sociais.

Uma segunda perspectiva de desenvolvimento da MEE está relacionada à associação da MEE com a psicometria moderna, a teoria de resposta ao item (TRI). Há uma grande semelhança de concepções da MEE e da TRI, pois as duas partem do princípio de traços latentes. A concatenação das duas técnicas é de grande importância para o desenvolvimento de ambas, à medida que permitem agregação mútua. A TRI trabalha com o desenvolvimento de instrumentos mais adequados aos princípios de medição das habilidades e características latentes humanas, enquanto a MEE necessita de instrumentos realmente eficazes para a mensuração de fenômenos latentes. A partir da construção de bancos de itens para a elaboração de instrumentos mais adequados, a MEE pode aprimorar seus modelos de mensuração, o que beneficiaria o desenvolvimento de modelos teóricos relevantes e eficientes para a descrição e explicação de fenômenos humanos e sociais. Já podem ser observados na literatura trabalhos (e.g.: Raju, Lafitte \& Byrne, 2002; Reise, Widaman \& Pugh, 1993) que buscam coadunar as duas técnicas de análise de dados e desenvolvimento de instrumentos, o que pode trazer notáveis benefícios para o desenvolvimento teórico das diversas áreas do saber.

Como toda técnica não muito difundida na comunidade científica, a MEE também merece atenção de pesquisadores para que problemas encontrados em decorrência do processo do desenvolvimento da pesquisa e análise dos dados possam ser evitados. $\mathrm{O}$ emprego da terminologia adequada para os achados resultantes de análises de MEE, bem como a clareza sobre limitações e características das possíveis conclusões, é de extrema importância para o bom andamento da produção científica. Em especial, aqueles mais "novos" no processo de produção de conhecimento, ou aqueles que se encontram no processo de formação como pesquisadores, podem ser contaminados pelo uso de conceitos sedutores no seio da MEE. Vários desses conceitos foram discutidos ao longo deste artigo, como da questão confirmatória de análise de dados e do estabelecimento de relações causais. Para o bom andamento da atividade científica é fundamental que potencialidades e limitações de técnicas de análise de dados sejam largamente e amplamente discutidas nos veículos de divulgação científica, propiciando que um debate sóbrio e eficiente seja realizado para auxiliar na produção do conhecimento em diferentes áreas do saber. Cabe ao pesquisador usuário da MEE tomar os devidos cuidados no processo de redação de relatos científicos que fazem uso da técnica, para que o desenvolvimento teórico daí decorrente não seja prejudicado pelo mal uso de conceitos relativos à MEE.

Tem-se como perspectiva que este trabalho seja um instigador dos pesquisadores para que busquem a diversificação de suas técnicas de análise de dados, mas que o façam com cuidado e zelo pelos aspectos teóricos e conceituais relativos ao uso de tais técnicas. A grande profusão de programas de análise de dados e computadores mais eficientes no processamento de grandes conjuntos de informações devem ser aproveitada em toda sua potencialidade, para que a capilarização de métodos e técnicas de produção de conhecimento sejam cada vez mais difundidos na ciência brasileira, propiciando a produção sólida e continuada de conhecimento sobre a realidade social e humana de nosso país.

\section{Referências}

Anastasi, A. \& Urbina, S. (2000). Testagem psicológica. Porto Alegre: Artmed.

Arbuckle, J. L. \& Worthke, W. (1999). AMOS 4.0 users guide. Chicago: SmallWaters.

Bollen, K. A. (1989). Structural equations with latent variables. New York: John Wiley and Sons.

Boomsma, A. (2000). Reporting analyses of covariance structures. Structural Equation Modeling, 7(3), 461-483.

Bullock, H. E., Harlow, L. L. \& Mulaik, S. A. (1994). Causation issues in structural equation modeling research. Structural Equation Modeling, 1(3), 253-267.

Byrne, B. (2001). Structural equation modeling with Amos: Basic concepts, applications and programming. New Jersey: Lawrence Erlbaum Associates.

Foguet, J. M. B. \& Gallart, G. C. (2001). Modelos de ecuaciones estructurales. Madrid: La Muralla.

Frederico, E. \& Robic, A. R. (2005). Determinantes da continuidade do relacionamento entre varejistas de moda e seus fornecedores: Uma comparação entre a utilização de modelos estruturais $e$ análise multivariada convencional [Trabalho completo]. Em ANDAP (Org.), Anais do XXIX ENANPAD (pp. 1-16). Brasília: ANPAD.

Gouveia, V. V., Martínez, E., Meira, M. \& Milfont, T. L. (2001). A estrutura e conteúdo universais dos valores humanos: análise fatorial confirmatória da tipologia de Schwartz. Estudos de Psicologia, 6(2), 133-142.

Hair, J. F., Anderson, R. E., Tatham, R. L. \& Black, W. C. (2005). Análise multivariada de dados. Porto Alegre: Artmed.

Hancock, G. R. \& Freeman, M. J. (2001). Power and sample size for the root mean square error of approximation test of not close fit in structural equation modeling. Educational and Psychological Measurement, 61(5), 741-758.

Hox, J. J. \& Bechger, T. M. (1998). An introduction to structural equation modeling. Family Science Review, 11, 354-373.

Kaplan, D. (2000). Structural Equation Modeling: foundations and extensions. Sage: Thousand Oaks.

Klem, L. (2002). Structural equation modeling. Em L. G. Grimm \& P. R. Yarnold (Orgs.), Reading and understanding more multivariate statistics (pp. 227-260). Washington: American Psychological Association.

Kline, R. B. (1998). Principles and practice of structural equation modeling. New York: Guilford Press.

Kozlowski, S. W. J. \& Klein, K. J. (2000). A multilevel approach to theory and research in organizations: contextual, temporal and emergent processes. Em K. J. Klein \& S. W. J. Kozlowski (Orgs.), Multilevel theory, research and methods in organizations (pp. 3-90). San Francisco: Jossey-Bass.

Laros, J. A. (2005). O uso de análise fatorial: algumas diretrizes para pesquisadores. Em L. Pasquali (Org.), Análise fatorial para pesquisadores (pp. 163-184). Brasília: LabPAM. 
Little, R. J. A. \& Rubin, D. B. (1987). Statistical analysis with missing data. New York: Lawrence Erlbaum.

MacCallum, R. C., Browne, M. W. \& Sugawara, H. M. (1996). Power analysis and determination of simple size for covariance structure modeling. Psychological Methods, 1, 130-149.

Mardia, K. V. (1971). The effect of nonnormality on some multivariate tests and robustness to nonnormality in the linear model. Biometrika, 58 (1), 105-121.

McDonald, R. P. \& Ho, M. R. (2002). Principles and practice in reporting structural equation analyses. Psychological Methods, 7(1), 64-82.

Medeiros, C. A. F., Albuquerque, L. G. \& Marques, G. M. (2005). Comprometimento organizacional: Um estudo de suas relações com características organizacionais e desempenho de empresas hoteleiras [Trabalho completo]. Em ANDAP (Org.), Anais do XXIX ENANPAD (pp. 1-16). Brasília: ANPAD.

Nunnally, J. C. \& Bernstein, I. H. (1994). Psychometric Theory (3a ed.). NewYork: McGraw-Hill.

Osborne, J. (2002). Notes on the use of data transformations. Practical Assessment, Research \& Evaluation [On-Line], 8(6). Retirado em 25/04/2003 do http:// ericae.net/pare/getvn. asp? $\mathrm{v}=8 \& \mathrm{n}=6$.

Pasquali, L. (2003). Psicometria. São Paulo: Vozes.

Pilati, R. \& Abbad, G. (2005). Análise fatorial confirmatória da escala de impacto do treinamento no trabalho. Psicologia: Teoria e Pesquisa, 21(1), 43-51.

Popper, K. (1972). A lógica da pesquisa científica. São Paulo: Cultrix.

Raju, N. S., Laffitte, L. J. \& Byrne, B. M. (2002). Measurement equivalence: A comparison of methods based on confirmatory factor analysis and item response theory. Journal of Applied Psychology, 87(3), 517-529.
Reise, S. P., Widaman, K. F. \& Pugh, R. H. (1993). Confirmatory factor analysis and item response theory: Two approaches for exploring measurement invariance. Psychological Bulletin, 114(3), 552-566.

Shadish, W. R., Cook, T. D. \& Campbell, D. T. (2002). Experimental and quasi-experimental design for generalized causal inference. Boston: Houghton Mifflin Company.

Tabachnick, B. G. \& Fidell, L. S. (2007). Using multivariate statistics ( $5^{\mathrm{a}}$ ed.). Boston: Pearson Education.

Thompson, B. (2002). Ten commandments of structural equation modeling. Em L. G. Grimm \& P. R. Yarnold (Orgs.), Reading and understanding more multivariate statistics (pp. 261-283). Washington: American Psychological Association.

Thompson, B. (2004). Exploratory and confirmatory factor analysis: understanding concepts and applications. Washington: American Psychological Association.

Ullman, J. B. (2007). Structural Equation Modeling. Em B. G. Tabachnick \& L. S. Fidell (Orgs.), Using multivariate statistics ( $5^{\mathrm{a}}$ ed.). Boston: Pearson Education.

Willms, J. D. (1999). Basic concepts in hierarchical linear modeling with applications for policy analysis. Handbook of Educational Policy. New York: Academic Press.

\section{CONGRESSO BRASILEIRO DE PSICOLOGIA DO DESENVOLVIMENTO}

Tema: Psicologia do Desenvolvimento e suas Interfaces com a Educação e a Saúde

Recebido em 14.12.2006

Primeira decisão editorial em 06.09.06

Versão final em 27.10.2006

Aceito em 12.12.2006

Local: Vitória - ES

Período: 06 a 09 de setembro de 2007

Realização:

Universidade Federal do Espírito Santo

Programa de Pós-graduação em Psicologia

Informações e inscrição:

www.sbpd.org.br 